\title{
Long non-coding RNA cancer susceptibility candidate 2 (CASC2) alleviates the high glucose-induced injury of CIHP-1 cells via regulating miR-9-5p/PPAR $\gamma$ axis in diabetes nephropathy
}

\author{
Feng $\mathrm{Li}^{1}$, Bo Dai ${ }^{2}$ and Xiquan $\mathrm{Ni}^{3^{*}}$
}

\begin{abstract}
Background: High glucose $(\mathrm{HG})$ induced podocytes injury plays an important role in diabetes nephropathy (DN) development. Long noncoding RNA cancer susceptibility candidate 2 (CASC2) was found to be decreased in serum of DN patients. We aimed to explore the function and possible mechanism of CASC2 in HG induced podocytes injury.

Methods: Under normal glucose (NG), HG and mannitol stimulated podocyte conditions, the levels of CASC2, microRNA-9-5p (miR-9-5p) and peroxisome proliferator-activated receptor gamma (PPAR $\gamma$ ) were examined by quantitative real-time polymerase chain reaction (qRT-PCR). Podocyte injury was evaluated by measuring cell viability and apoptosis of CIHP-1 cells were checked by cell counting kit-8 (CCK-8) assay and flow cytometry, respectively. Western blot was used to detect all protein levels. Dual-luciferase reporter, RNA immunoprecipitation (RIP) and RNA pull-down assays were performed to confirm the relationship between CASC2 and miR-9-5p.

Results: HG stimulation inhibited the expression levels of CASC2 and PPARy, but promoted the expression of miR9-5p. HG could restrain cell viability, autophagy and facilitate apoptosis in CIHP-1 cells, while CASC2 overexpression could reverse HG-induced podocytes injury. Furthermore, CASC2 could be used as a ceRNA to adsorb miR-9-5p, and miR-9-5p mimic overturned the effects of CASC2 on cell viability, autophagy and apoptosis in HG-stimulated podocytes. Additionally, PPAR $y$ was a target gene of miR-9-5p, and CASC2 could weaken the HG-induced podocytes injury by up-regulating PPARY.
\end{abstract}

Conclusion: CASC2 increased cell viability, autophagy and inhibited cell apoptosis by regulating miR-9-5p/PPARY axis, thus reducing the HG-induced podocytes injury.

Keywords: High glucose, Podocyte, CASC2, miR-9-5p, PPARy

*Correspondence: gltxs009@163.com

${ }^{3}$ Department of Nephrology, Tai'an Campus of the 960th Hospital

of the Chinese People's Liberation Army, No.217 Huanshan Road, Taishan District, Tai'an 271000, Shandong, China

Full list of author information is available at the end of the article

\section{Background}

Diabetes is a common endocrine disease, among which the prevalence of diabetes nephropathy (DN) is $20-40 \%$ [1]. It is estimated that the number of DN patients is expected to increase to 642 million by 2040 [2]. DN is characterized by the presence of albuminuria and a decreased glomerular filtration rate [3]. Podocyte cells (podocytes) are epithelial cells in the 
visceral layer of renal follicles, which play a key role in the pathogenesis of DN and are an important component of glomerular filtration barrier $[4,5]$. Several studies have revealed the correlation between podocytes injury (death and apoptosis) and albuminuria [6], and reducing podocyte injury can improve DN [7]. However, the mechanism for alleviating podocytes injury remains unclear.

Long non-coding RNAs (lncRNAs), are non-proteincoding RNA molecules longer than 200 nucleotides, which are widely regarded as the important regulators in cellular function and disease processes [8]. Increased evidences suggested that lncRNA could modulate DN progression. For instance, lncRNAs GM5524 and GM15645 could regulate the HG-stimulated podocyte autophagy in DN [9]. LncRNA PVT1 knockdown repressed podocytes injury and apoptosis via increasing FOXA1 [10]. However, there are still many lncRNAs in DN function and molecular mechanisms have not been studied.

LncRNA cancer susceptibility candidate 2 (CASC2), located on chromosome 10q26, plays a regulatory role as an anti-cancer factor in various cancers, such as hepatocellular carcinoma [11] and pancreatic carcinoma [12]. Recently, Wang et al. revealed that CASC2 was specifically reduced in serum and renal tissues of type 2 diabetes patients with chronic renal failure, and follow-up identified that the serum of patients with low CASC2 expression had higher incidence of chronic renal failure [13]. MicroRNA-9-5p (miR-9-5p) is both a tumor depressor and a tumor promoter [14, 15]. A report demonstrated that miR-9-5p was related to complications of nephropathy in Type 1 and Type 2 diabetes patients [16]. The mechanism by which lncRNA can serve as the competing endogenous RNA (ceRNA) for miRNA to modulate the abundance of mRNA has been widely reported [17]. Peroxisome proliferator-activated receptor gamma (PPAR $\gamma$ ) is implicated in several metabolic syndromes, including DN. Down-regulated PPAR $\gamma$ could activate $\beta$-catenin signaling to destroy podocyte architectural integrity and increase cell apoptosis in DN [18]. Furthermore, lncRNA TUG1 could relieve extracellular matrix accumulation by sponging miR-377 and regulating PPARY in DN [19]. Based on the above findings, we speculated whether CASC2 can modulate PPAR $\gamma$ expression by serving as a ceRNA of miR-9-5p in DN.

In this work, we aimed to explore the effects of CASC2 on cell viability, apoptosis and autophagy in high glucose (HG) induced podocytes, and probe the relationship among CASC2, miR-9-5p and PPAR $\gamma$, providing a new perspective on the molecular mechanism of podocytes injury in $\mathrm{DN}$.

\section{Materials and methods}

\section{Cell culture and high glucose induction}

Human podocytes CIHP-1 (Ximbio, London, USA) were cultured in a Dulbecco's modified Eagle's medium (DMEM, Invitrogen, Carlsbad, CA, USA) containing 10\% fetal bovine serum (FBS, Gibco, Carlsbad, CA, USA) at a temperature of $37{ }^{\circ} \mathrm{C}$ with $5 \% \mathrm{CO}_{2}$. When cells density reached about 70\%, CIHP-1 cells were exposed to normal glucose (NG, $5 \mathrm{mM}$ ), high glucose (HG, $30 \mathrm{mM}$ ) or mannitol $(30 \mathrm{mM})$, and the exposure time was determined by individual experiments required.

\section{Cell transfection}

CASC2 overexpressed plasmid (CASC2) and its control Vector, small interfering RNAs against CASC2 and PPARY (si-CASC2, si-PPARY) and matched si-NC were provided by GenePharma (Shanghai, China). miR-9-5p mimic (miR-9-5p), inhibitor (anti-miR-9-5p) and their corresponding references (miR-NC, anti-NC) were synthesized by Beyotime (Beijing, China). Transfection of podocytes was performed by using Lipofectamine 3000 (Invitrogen).

\section{Quantitative real-time polymerase chain reaction (qRT-PCR)}

The RNA in CIHP-1 cells was extracted by TRIzol (Invitrogen), and the complementary DNA (cDNA) was synthesized via reverse transcription using HiScript Q RT Super Mix (Vazyme, Piscataway, NJ, USA). The reverse transcription was performed at $42{ }^{\circ} \mathrm{C}$ for $10 \mathrm{~min}$ and at $85^{\circ} \mathrm{C}$ for $10 \mathrm{~s}$. qRT-PCR analysis was conducted on 7500 Real-Time PCR System (Applied Biosystems, Foster City, CA, USA) using the SYBR premix Ex TaqIIkit (TaKaRa, Wuhan, China). Glyceraldehyde-3-phosphate dehydrogenase (GAPDH) and U6 were used as endogenous controls for CASC2/PPAR $\gamma$ and miR-9-5p, respectively. The primers used in this paper were synthesized by GenePharma and the sequences were used as below: CASC2, forward (F) 5'-GCACATTGGACGGTGTTTCC-3', reverse (R) 5'-CCCAGTCCTTCACAGGTCAC-3'. miR9-5p, F 5'-GTGCAGGGTCCGAGGT-3', R 5'-GCGCTC TTTGGTTATCTAGC-3'. PPAR $\gamma$, F 5'-AGAGCCTTC CAACTCCCTCA-3', R $5^{\prime}$-AACAGCTTCTCCTTC TCGGC-3'. U6, F 5'-TTGGTGCTCGCTTCGGCA-3', R 5'-GTGCAGGGTCCGAGGT-3'. GAPDH, F 5'-GGA GTCCACTGGTGTCTTCA-3', R 5'-GGGAACTGA GCAATTGGTGG-3'.

\section{Cell viability and apoptosis detection}

CIHP-1 cells were tiled into the 96-well plates and exposed to different treatments (HG, NG, HG+Vector, $\mathrm{HG}+\mathrm{CASC} 2$ and so on). At given points in time (12 h, $24 \mathrm{~h}$ and $48 \mathrm{~h}$ ), $10 \mu \mathrm{L}$ cell counting kit-8 (CCK-8, 
Beyotime) was added to the cells and cultured for another $2 \mathrm{~h}$ at $37^{\circ} \mathrm{C}$. Finally, the absorbance at $450 \mathrm{~nm}$ was measured by Biotek-Epoch2 (Beijing, China).

The apoptosis of podocytes CIHP-1 was estimated at $48 \mathrm{~h}$ after exposure to different treatments by using an Annexin V fluorescein isothiocyanate (FITC) and propidium iodide (PI) apoptosis detection kit (Keygen, Beijing, China). Briefly, podocytes were collected and were then suspended in $5 \mu \mathrm{L}$ FITC and $5 \mu \mathrm{L}$ PI in the absence of light for $15 \mathrm{~min}$. The apoptosis of CIHP-1 cells was checked by a flow cytometer (BD Biosciences, Franklin Lake, NJ, USA)

\section{Western blot assay}

Total protein from CIHP-1 cells was extracted by RIPA (Beyotime), and denatured at $98^{\circ} \mathrm{C}$ for 7 min before separation, and then transferred to polyvinylidene difluoride (PVDF, Beyotime) membranes. Membranes were sealed with $5 \%$ milk for $2 \mathrm{~h}$ before incubation with primary antibodies against B-cell lymphoma-2 (BCL-2, 1:1000, Abcam, Cambridge, MA, USA), Cleaved-caspase-3 (1:500, Abcam), Light chain 3-II (LC3-II, 1:3000, Abcam), LC3-I (1:1000, Abcam), Beclin 1 (1:2000, Beyotime), PPAR $\gamma$ (1:500, Abcam) or GAPDH (1:2000, Beyotime) overnight at $4{ }^{\circ} \mathrm{C}$. HRP-conjugated secondary antibody (1:4000, Abcam) was employed to incubate the membranes for another $1 \mathrm{~h}$. And the proteins were visualized by using BeyoECL Moon (Beyotime).

\section{Dual-luciferase reporter assay}

CASC2 wild type (CASC2-wt) with miR-9-5p binding sites and its mutant type (CASC2-mut) without binding sites were co-transfected into CIHP-1 cells with miR9-5p or miR-NC, respectively. Transfection was continued for $36 \mathrm{~h}$ and luciferase activity was evaluated through a Dual-luciferase reporter kit (Promega, Madison, WI, USA). In the same manner, PPAR $3^{\prime}$ untranslated region (3'UTR)-wt with miR-9-5p binding sites and PPARy 3'UTR-mut were co-transfected into cells with miR-9-5p or miR-NC, respectively, and the luciferase activity was detected.

\section{RNA immunoprecipitation (RIP) assay and RNA pull-down assay}

RIP detection was conducted using a Magna RIP RNABinding Protein Immunoprecipitation Kit (Millipore, Billerica, MA, USA). CIHP-1 cells were treated with miR9-5p or miR-NC, 48 h later, cells were lysed in RIP Lysis Buffer containing protease inhibitors. Then, Argonaute2 (Ago2) or ImmunoglobulinG (IgG) antibody (Abcam) were added to the cell lysates overnight at $4{ }^{\circ} \mathrm{C}$, and the immunoprecipitated RNAs were obtained, CASC2 and miR-9-5p levels were estimated using qRT-PCR analysis.
CIHP-1 cells were transfected with Biotin labeled BiomiR-9-5p and Bio-miR-NC, respectively. At $48 \mathrm{~h}$ posttransfection, cells were collected and the bound RNA was obtained by using a Pierce ${ }^{\mathrm{TM}}$ Magnetic RNA Pull-Down Kit (Thermo Fisher Scientific, Waltham, MA, USA) according to the instructions. Finally, CASC2 enrichment was assessed by qRT-PCR.

\section{Statistical analysis}

Data were acquired from at least three independent repetitions and displayed as mean \pm standard deviation (SD). Difference analysis was conducted by Student's $t$-test with two groups and one-way analysis of variance (ANOVA) with multiple groups using GraphPad Prism 8 . The $P$ value less than 0.05 was regarded as statistically distinct.

\section{Results}

\section{CASC2 alleviated the HG-induced podocytes injury}

Firstly, we examined the expression of CASC2 in human podocytes treated with NG, HG or mannitol by qRTPCR. The results showed that HG significantly decreased CASC2 expression in CIHP-1 cells compared with NG and mannitol treatment (Fig. 1a). In addition, a timedependent reduction in CASC2 expression was displayed in HG-treated CIHP-1 cells (12, 24 and 48 h) (Fig. 1b). In view of the expression of CASC2 was substantially reduced at $48 \mathrm{~h}$ of $\mathrm{HG}$ stimulation, we then overexpressed CASC2 in HG-stimulated CIHP-1 cells for $48 \mathrm{~h}$, and overexpression efficiency was identified by qRT-PCR. As shown in Fig. 1c, CASC2 expression was obviously promoted in HG-stimulated CIHP-1 cells after transfection of CASC2 for $48 \mathrm{~h}$. CCK- 8 and flow cytometry results indicated that overexpression of $\mathrm{CASC} 2$ induced cell viability (Fig. 1d) and retarded apoptosis (Fig. 1e) in HGtreated CIHP-1 cells. To confirm the results of apoptosis, we detected the expression of apoptosis marker proteins BCL-2 and Cleaved-caspase-3. Western blot assay demonstrated that up-regulation of CASC2 enhanced BCL-2 expression and silenced Cleaved-caspase- 3 expression (Fig. 1f), which was in agreement with the results of Annexin V-FITC/PI. Furthermore, HG could reduce the ratio of LC3-II/LC3-I and Beclin 1 expression in CIHP-1 cells, and CASC2 overexpression reversed the effects of $\mathrm{HG}$ on the expression of autophagy related proteins (Fig. 1g). The above findings indicated that CASC2 could alleviate the HG-induced podocytes injury by affecting cell viability, apoptosis and autophagy.

\section{CASC2 directly interacted with miR-9-5p}

LncRNA generally functions as a sponge for miRNA in human diseases [20]. We speculated whether CASC2 could also act as miRNA sponge to regulate 


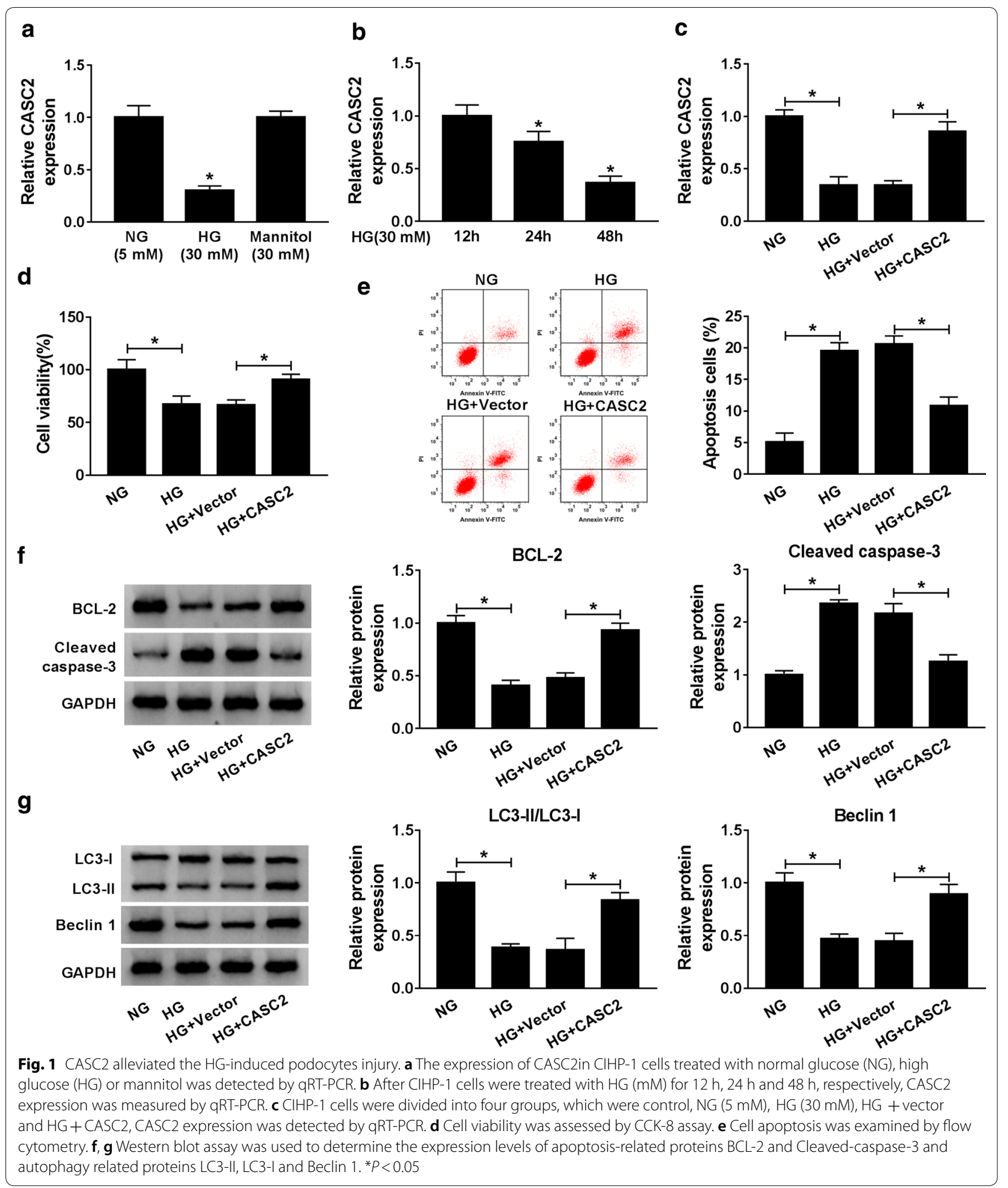


HG-induced podocytes injury. As shown in Fig. 2a, we found that miR-9-5p was up-regulated in HGtreated CIHP-1 cells compared to cells treated with NG or mannitol, and miR-9-5p expression was drastically augmented in HG-treated CIHP-1 cells in a timedependent manner (Fig. 2b). Interestingly, there were complementary sites between miR-9-5p and CASC2 by bioinformatics website starBase v2.0 (Fig. 2c). Dual-luciferase reporter assay showed that the luciferase activity of CASC2-wt was obviously decreased in CIHP-1 cells transfected with miR-9-5p than that cells transfected with miR-NC, whereas, it was no significant difference in luciferase activity of CASC2-mut (Fig. 2d). RIP assay indicated that the enrichments of CASC2 and miR-9-5p were higher in CIHP-1 cells incubated with Ago2 (Fig. 2e). RNA pull-down assay further revealed that the enrichment of CASC2 in Bio-miR-9-5p group was aggrandized relative to that Bio-NC group (Fig. $2 \mathrm{f}$ ). These results strongly supported that CASC2 could specifically bind to miR-9-5p. Meanwhile, qRT-PCR data showed that CASC2 knockdown in CIHP-1 cells elevated miR-9-5p expression, and CASC2 overexpression degraded miR-9-5p expression (Fig. 2g, h). These results suggested that CASC2 could act as a ceRNA to negatively regulated miR-9-5p expression in podocytes.

\section{CASC2 regulated the HG-induced podocytes injury via targeting miR-9-5p}

As presented in Fig. 3a, miR-9-5p mimic (miR-9-5p) could reverse the inhibitory effect of CASC2 overexpression on miR-9-5p expression in HG-induced CIHP-1 cells. As expected, the impact of CASC2 on promoting cell activity (Fig. 3b) and inhibiting cell apoptosis (Fig. 3c) in HG-stimulated CIHP-1 cells was offset by miR-9-5p. Simultaneously, the inhibition of CASC2 on the protein expression of Cleaved-caspase- 3 and the promotion of CASC2 on LC3-II/LC3-I ratio as well as the levels of BCL-2 and Beclin 1 could be weakened by transfection of miR-9-5p in HG-induced CIHP-1 cells (Fig. 3d, e). The obtained data proved that CASC2 attenuated the HGinduced podocytes injury by down-regulating miR-9-5p.

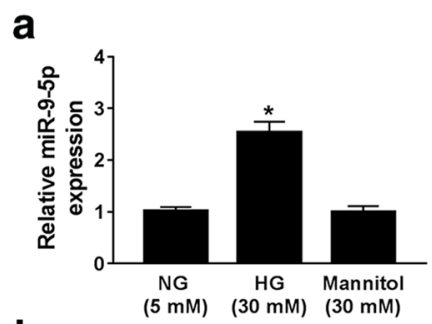

b

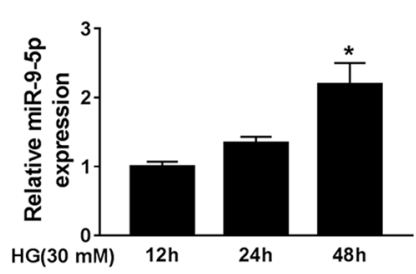

f

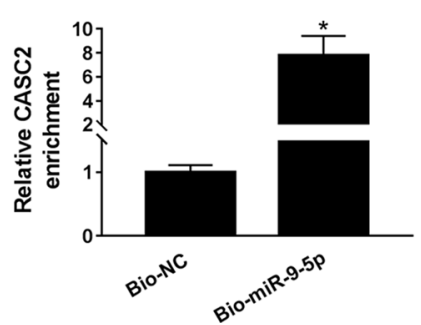

C

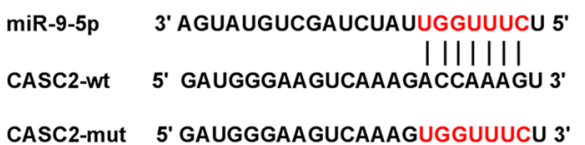

d
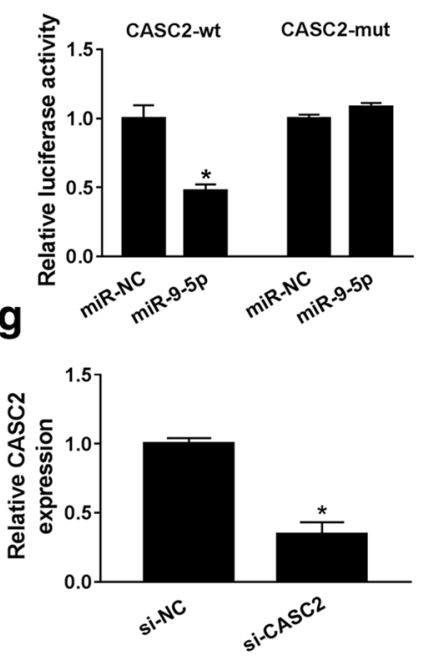

e

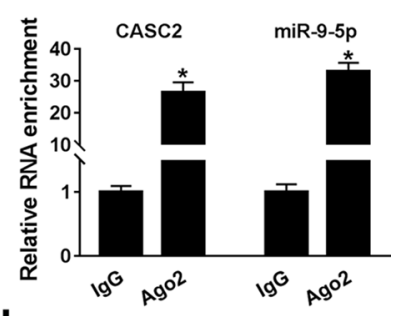

h

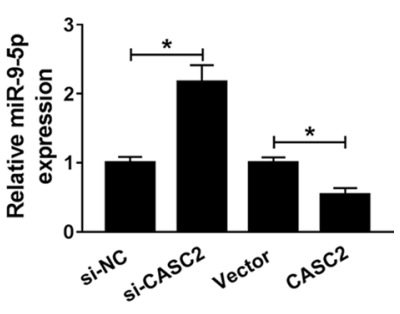

Fig. 2 CASC2 directly interacted with miR-9-5p. a The expression of miR-9-5pin CIHP-1 cells treated with normal glucose (NG), high glucose (HG) or mannitol was measured by qRT-PCR. b After CIHP-1 cells were treated with HG (mM) for $12 \mathrm{~h}, 24 \mathrm{~h}$ and $48 \mathrm{~h}$, respectively, miR-9-5p expression was examined by qRT-PCR. c StarBase v2.0 was used to predict the target miRNAs of CASC2. $\mathbf{d}$-f Dual luciferase reporter, RIP and RNA pull-down assays were utilized to assess the combination of CASC2 and miR-9-5p. g CASC2 expression in CIHP-1 cells transfected with si-NC or si-CASC2 was determined by qRT-PCR. $\mathbf{h}$ The expression of miR-9-5 pin CIHP-1 cells transfected with si-NC, si-CASC2, Vector or CASC2 was measured using qRT-PCR analysis. ${ }^{*} P<0.05$ 


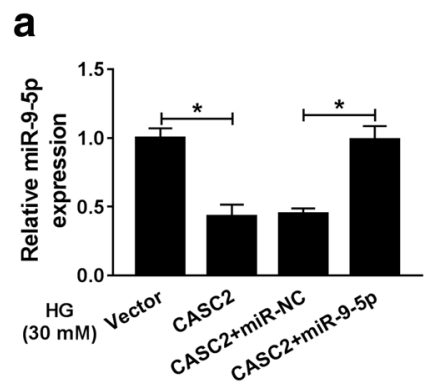

d

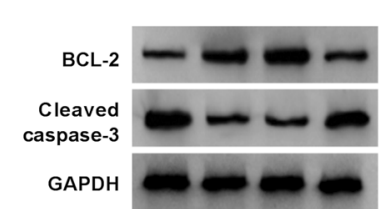

$\mathbf{e}$
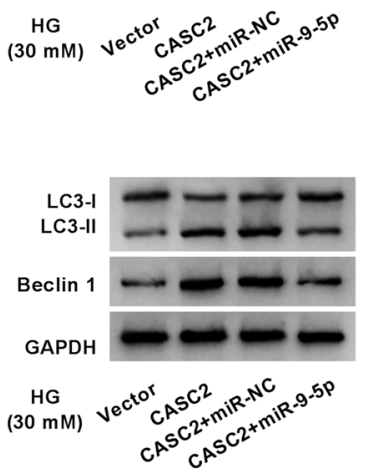

b

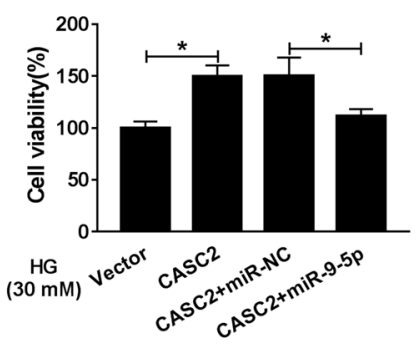

BCL-2

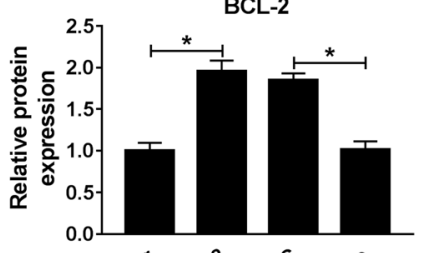

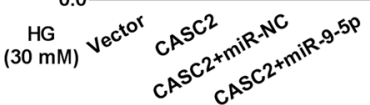

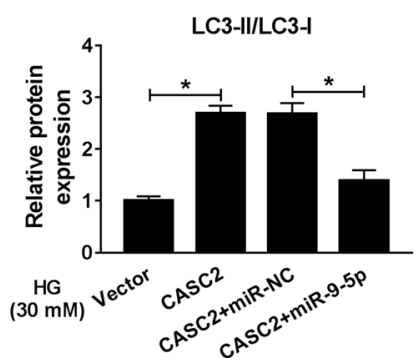

C

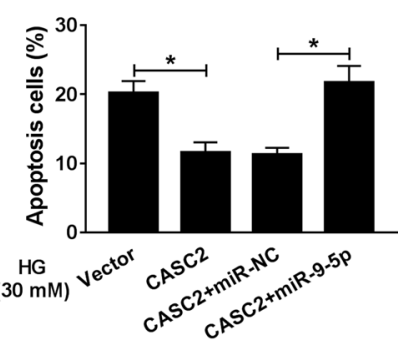

Cleaved caspase-3

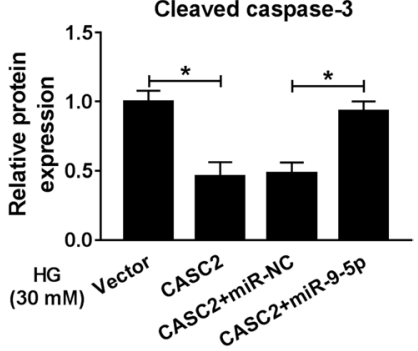

Beclin 1

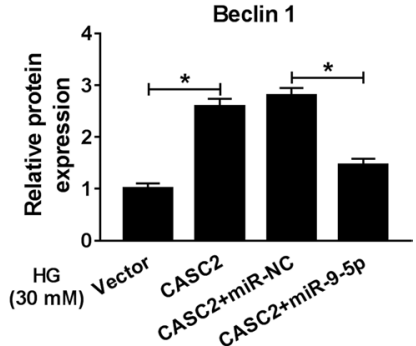

Fig. 3 CASC2 regulated the HG-induced podocytes injury via targeting miR-9-5p. The HG-treated CIHP-1 cells were divided into four groups: Vector, CASC2, CASC2 + miR-NC and HG + miR-9-5p. a The expression of miR-9-5p was examined by qRT-PCR. b, c Cell viability and apoptosis were evaluated by CCK-8 assay and flow cytometry, respectively. $\mathbf{d}$, e The expression levels of apoptosis-related proteins BCL-2 and Cleaved-caspase-3 and autophagy related proteins LC3-II, LC3-I and Beclin 1 were detected by western blot assay. ${ }^{*} P<0.05$

\section{CASC2 acted as a ceRNA by sponging miR-9-5p to facilitate} PPARY expression

As appeared in Fig. 4a-d, HG inhibited the mRNA and protein levels of PPAR $\gamma$ in CIHP-1 cells compared to $\mathrm{NG}$ and mannitol stimulation. At $48 \mathrm{~h}$ after the induction of HG, the mRNA and protein levels of PPAR $\gamma$ were dwindled in CIHP-1 cells. The effect of HG treatment on PPARy expression was the opposite of that of miR9-5p, thus we speculated whether there was a connection between miR-9-5p and PPAR $\gamma$. As presented in Fig. 4e, there were binding sites for miR-9-5p in the 3'UTR of PPAR $\gamma$. Dual-luciferase reporter assay showed that miR9-5p markedly decreased the luciferase activity of PPAR $\gamma$ 3'UTR-wt in CIHP-1 cells than that PPAR $\gamma$ 3'UTR-mut (Fig. 4f), suggesting PPAR $\gamma$ was the target mRNA of miR-9-5p. Then, we examined the effect of miR-9-5p on PPAR $\gamma$ expression, the interference efficiency of antimiR-9-5p on miR-9-5p expression was first examined by qRT-PCR (Fig. 4g). Western blot data showed that the overexpressed miR-9-5p could restrain the protein expression of PPAR $\gamma$, while the decreased miR-9-5p could raise PPAR y protein expression (Fig. 4h). Additionally, we found that CASC2 depletion reduced the protein expression of PPAR $\gamma$, and co-transfection of anti-miR9-5p could reverse this effect (Fig. 4i). The above findings revealed that CASC2 positively regulated PPAR $\gamma$ expression by acting as a ceRNA for miR-9-5p in podocytes.

\section{CASC2 alleviated the HG-induced podocytes injury by increasing PPARY}

Considering CASC2 could act as a sponge of miR-9-5p to regulate the expression of PPAR $\gamma$, we further investigated whether PPAR $\gamma$ was involved in regulation of HG-induced podocytes injury mediated by CASC2. Western blot results indicated that co-transfection of si-PPAR $\gamma$ neutralized the promoting effect of CASC2 on PPARy protein expression (Fig. 5a). The data of CCK-8 and Annexin V-FITC/PI assays indicated that the effects of CASC2 on cell viability (Fig. 5b) and apoptosis (Fig. 5c) could be abolished by silencing 

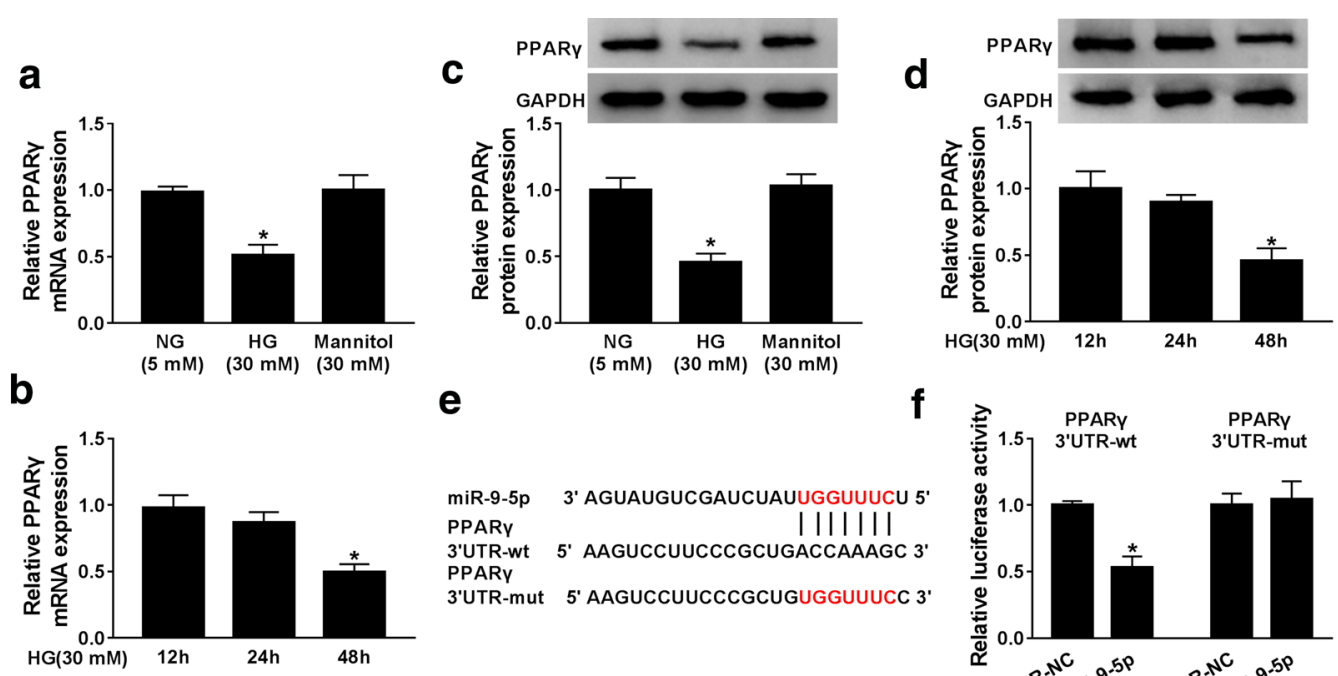

e
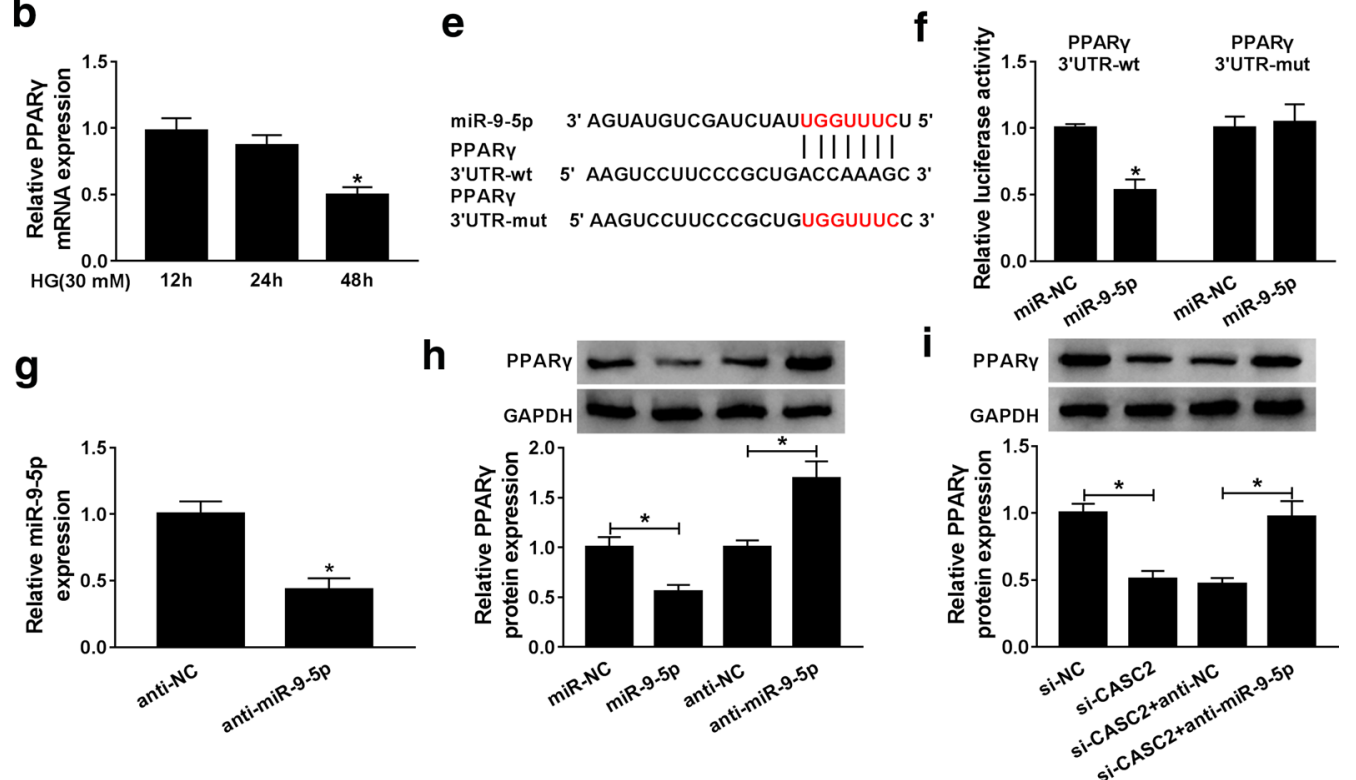

Fig. 4 CASC2 acted as a ceRNA by sponging miR-9-5p to facilitate PPARy expression. a The mRNA expression of PPARy in NG, HG or mannitol-treated CIHP-1 cells was analyzed by qRT-PCR. $\mathbf{b}$ qRT-PCR assay was used to measure the mRNA expression of PPARY in CIHP-1 cells treated by HG (30 mM) at different times. c The protein expression of PPARy in NG, HG or mannitol-treated CIHP-1 cells was analyzed by western blot assay. d Western blot assay was used to measure the protein expression of PPARy in CIHP-1 cells treated by HG (30 mM) at different times. e StarBase v2.0 predicted that there were binding sites between miR-9-5p and PPARY. f Dual luciferase reporter assay was conducted to detect the interaction between miR-9-5 $p$ and PPARy in CIHP-1 cells. $\mathbf{g}$ The expression of miR-9-5 $p$ in CIHP-1 cells transfected with anti-NC or anti-miR-9-5 $p$ was measured by qRT-PCR. $\mathbf{h}$ The protein expression of PPARy in CIHP-1 cells transfected with miR-NC, miR-9-5p, anti-NC or anti-miR-9-5p was assessed using western blot assay. i PPARY protein expression in CIHP-1 cells transfected with si-NC, si-CASC2, si-CASC2 + anti-NC, or si-CASC2 + anti-miR-9-5p was estimated by western blot. ${ }^{*} P<0.05$

PPAR $\gamma$ in HG-induced CIHP-1 cells. Similarly, the effects of CASC2 on levels of Cleaved-caspase-3, BCL2, Beclin 1 and LC3-II/LC3-I ratio were rescued by si-PPAR $\gamma$, implying PPAR $\gamma$ knockdown could increase Cleaved-caspase- 3 protein, and decrease the expression levels of BCL-2 and Beclin 1 as well as the ratio of LC3-II/LC3-I (Fig. 5d, e). To sum up, CASC2 alleviated the HG-induced podocytes injury by up-regulating PPAR $\gamma$.

Overall, it could be concluded that HG inhibited cell viability, autophagy but promoted cell apoptosis by downregulating CASC2 and PPAR $\gamma$ expression as well as upregulating miR-9-5p in CIHP-1 cells (Fig. 6).

\section{Discussion}

Podocytes are terminally differentiated visceral epithelial cells, which are important components of the glomerular filtration barrier. Podocyte viability and apoptosis as well as autophagy can affect glomerular function [21]. A large number of studies have shown that high glucose induction can cause podocytes injury [22-24].

Several lncRNAs, such as lncRNA MALAT1 [25] and lncRNA PRINS [26], have been found to be involved in the development of $\mathrm{DN}$, they regulated mRNA expression at the post-transcriptional level. In this study, we found that CASC2 expression was prominently downregulated in high glucose-stimulated podocytes in a 


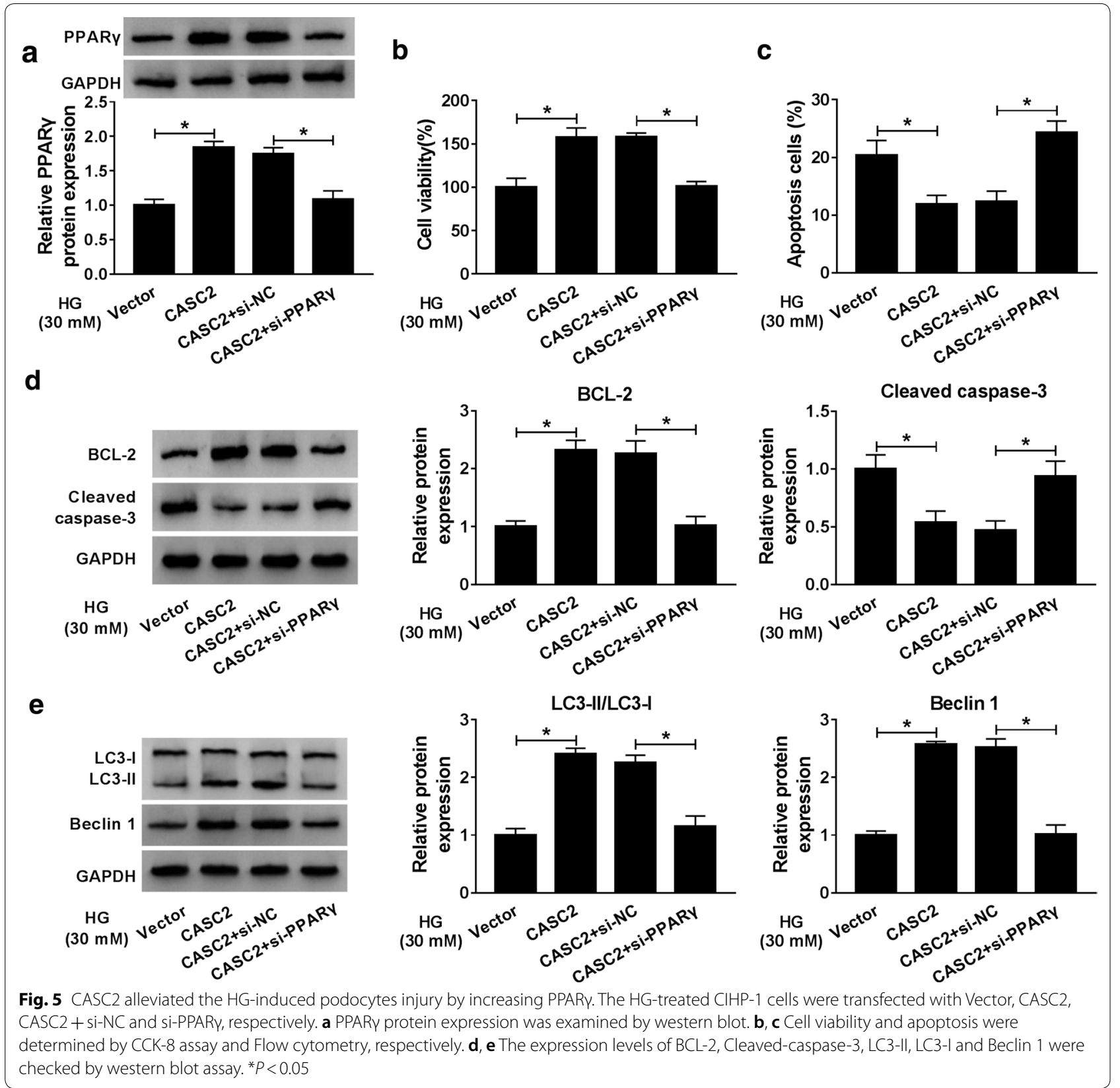

time-dependent manner and dose-response manner. Autophagy is a double-edged sword, and its excessive activation or repression can cause podocytes injury [27]. Autophagy activity is impaired in DN patients, so promoting autophagy to some extent can reduce podocytes injury [28], and Beclin 1, LC3-I and LC3-II have been shown to be autophagy specific proteins [29]. In accordance with previous data, high glucose could inhibit cell viability and autophagy, and promote cell apoptosis, while overexpression of CASC2 could attenuate the effect of high glucose on podocytes injury, suggesting the protective effect of CASC2 on podocytes injury. Similarly, Yang et al. observed that CASC2 was enormously decreased in DN patients, while there was no significant difference in CASC2 expression in DN patients as compared to those with DM without complication (DN) [30]. Besides, Wang et al. reported that the development of type 2 diabetes might have no significant effects on CASC2 expression in renal tissue, whereas CASC2 expression in renal tissues was found to be evidently lower in patients with type 2 diabetes complicated with chronic renal failure [13]. These data suggested that 


\section{CIHP-1 cell injury}
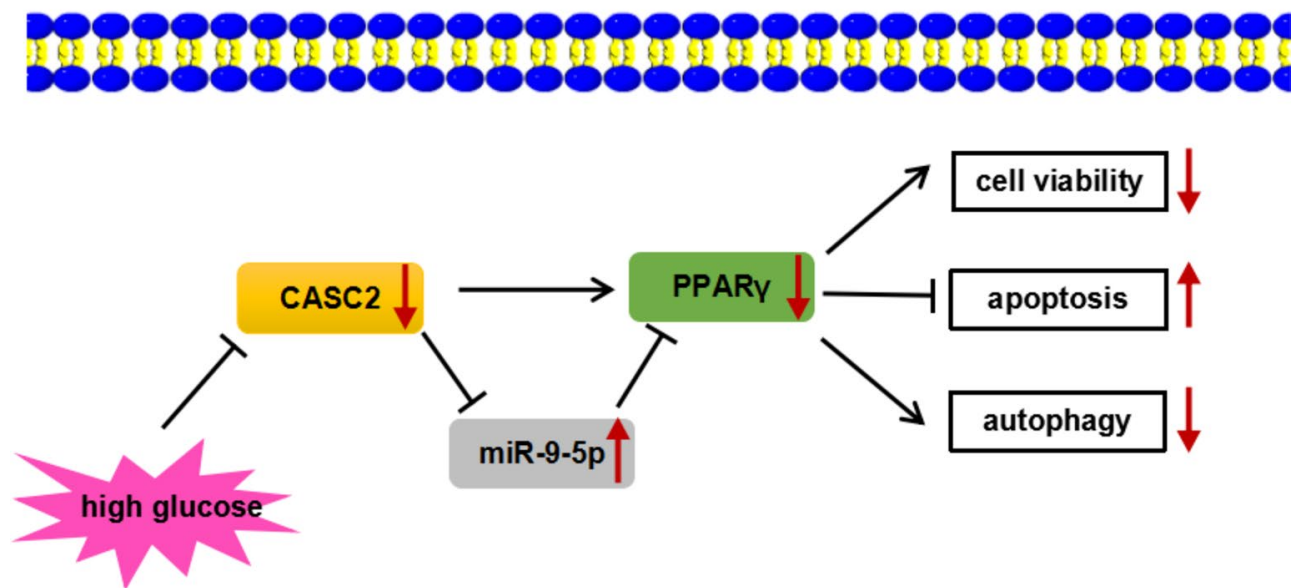

Fig. 6 Schema presented the mechanism that HG repressed cell viability, autophagy and promoted cell apoptosis by regulating the CASC2/ miR-9-5P/PPARY axis in CIHP-1 cells

CASC2 inhibition was very likely to be involved in the pathogenesis of DN. Moreover, lncRNA often functions as ceRNA, and we speculated that CASC2 might also be involved in the regulation of podocytes injury by sponging miRNA.

Compared with non-diabetic subjects, the level of miR9-5p was higher in serum of patients with gestational diabetes mellitus [31], and serum miR-9 might be an underlying marker for poor prognosis of DN [32]. In our data, the abundance of miR-9-5p was increased in high glucose-induced podocytes, and miR-9-5p was validated to be the target miRNA for CASC2, and CASC2 could inversely modulate miR-9-5p expression in podocytes. Recovery experiments showed that CASC2 mitigated podocytes injury by decreasing miR-9-5p via acting as a miR-9-5p sponge, similar to the work of Zhang et al., who indicated that IncRNA SOX2OT could reduce the high glucose-stimulated podocytes damage by autophagy induction through binding to miR-9 [33]. Therefore, it is reasonable to infer that CASC2 regulated podocyte activity, apoptosis and autophagy through sponging miR-9-5p.

PPAR $\gamma$ agonists have been widely reported to improve glycemic status in diabetes patients [34], and PPAR $\gamma$ has favorable renal protective effects [35]. As expected, high glucose treatment obviously retarded PPARy expression. Importantly, miR-9-5p directly targeted PPAR $\gamma$ 3'UTR and negatively modulated its expression. In addition, CASC2 could regulate PPAR $\gamma$ expression by sponging miR-9-5p, based on these results, we hypothesized whether CASC2 implicated in podocytes injury by regulating PPARy. The results showed that PPAR $\gamma$ knockdown neutralized the effect of CASC2 on podocytes injury. Besides, PPAR $\gamma$ has been shown to restore podocyte integrity to improve proteinuria [36].

\section{Conclusion}

In summary, we believed that CASC2 mainly up-regulated the expression of PPAR $\gamma$ by acting as the ceRNA of miR-9-5p, thus alleviating HG-induced podocytes injury through increasing cell viability, autophagy and reducing cell apoptosis. This study provided a new molecular regulatory mechanism for podocytes injury induced by HG in DN.

\section{Abbreviations}

HG: High glucose; DN: Diabetes nephropathy; CASC2: Cancer susceptibility candidate 2; NG: Normal glucose; RIP: RNA immunoprecipitation; PPARY: Peroxisome proliferator-activated receptor gamma.

\section{Acknowledgements}

The authors sincerely appreciate all members participated in this study.

\section{Authors' contributions}

$F L$ designed the experiments, performed the experiments, and analyzed and collected the data, wrote the manuscript. BD analyzed interpreted the data, performed the experiments and wrote the manuscript. XN performed the experiments and analyzed the data. All authors read and approved the final manuscript.

\section{Funding}

This work was supported by Key Research \& Development Program of Shandong Province, China [Grant No.2019GSF108172], Natural Science Foundation of Shandong Province, China [Grant No.2016ZRA08005] and Science and Technology Development Plans of TCM of Shandong Province, China [Grant No. 2015-443].

\section{Availability of data and materials}

The datasets used and/or analyzed during the current study are available from the corresponding author on reasonable request. 
Ethics approval and consent to participate

Not applicable.

\section{Patient consent for publication}

Not applicable.

\section{Competing interests}

The authors declare that they have no competing interest.

\section{Author details}

1 Department of Nephrology, Heze Mudan People's Hospital, Heze, Shandong, China. ${ }^{2}$ Department of Nephrology, Liaocheng People's Hospital, Liaocheng, Shandong, China. ${ }^{3}$ Department of Nephrology, Tai'an Campus of the 960th Hospital of the Chinese People's Liberation Army, No.217 Huanshan Road, Taishan District, Tai'an 271000, Shandong, China.

Received: 18 February 2020 Accepted: 27 July 2020

Published online: 06 August 2020

\section{References}

1. Dronavalli S, Duka I, Bakris GL. The pathogenesis of diabetic nephropathy. Nat Clin Pract Endocrinol Metab. 2008:4(8):444-52.

2. Ogurtsova K, da Rocha Fernandes JD, Huang Y, Linnenkamp U, Guariguata L, Cho NH, et al. IDF Diabetes Atlas: Global estimates for the prevalence of diabetes for 2015 and 2040. Diabetes Res Clin Pract. 2017;128:40-50.

3. Afkarian M, Zelnick LR, Hall YN, Heagerty PJ, Tuttle K, Weiss NS, et al. Clinical manifestations of kidney disease among US adults with diabetes, 1988-2014. JAMA. 2016;316(6):602-10.

4. Ziyadeh FN, Wolf G. Pathogenesis of the podocytopathy and proteinuria in diabetic glomerulopathy. Curr Diabetes Rev. 2008;4(1):39-45.

5. Berthier CC, Zhang H, Schin M, Henger A, Nelson RG, Yee B, et al. Enhanced expression of Janus kinase-signal transducer and activator of transcription pathway members in human diabetic nephropathy. Diabetes. 2009;58(2):469-77.

6. White KE, Bilous RW, Marshall SM, El Nahas M, Remuzzi G, Piras G, et al. Podocyte number in normotensive type 1 diabetic patients with albuminuria. Diabetes. 2002;51(10):3083-9.

7. Mathieson PW. The podocyte as a target for therapies-new and old. Nat Rev Nephrol. 2011;8(1):52-6.

8. Boon RA, Jae N, Holdt L, Dimmeler S. Long noncoding RNAs: from clinical genetics to therapeutic targets? J Am Coll Cardiol. 2016;67(10):1214-26.

9. Feng $Y$, Chen $S, X u$ J, Zhu Q, Ye X, Ding D, et al. Dysregulation of IncRNAs GM5524 and GM15645 involved in high glucose induced podocyte apoptosis and autophagy in diabetic nephropathy. Mol Med Rep. 2018;18(4):3657-64

10. Liu DW, Zhang JH, Liu FX, Wang XT, Pan SK, Jiang DK, et al. Silencing of long noncoding RNA PVT1 inhibits podocyte damage and apoptosis in diabetic nephropathy by upregulating FOXA1. Exp Mol Med. 2019:51(8):88.

11. Refai NS, Louka ML, Halim HY, Montasser I. Long non-coding RNAs (CASC2 and TUG1) in hepatocellular carcinoma: Clinical significance. J Gene Med. 2019;21(9):e3112.

12. Zhang $H$, Feng $X$, Zhang $M$, Liu A, Tian L, Bo W, et al. Long non-coding RNA CASC2 upregulates PTEN to suppress pancreatic carcinoma cell metastasis by downregulating miR-21. Cancer Cell Int. 2019;19:18.

13. Wang L, Su N, Zhang Y, Wang G. Clinical significance of serum IncRNA cancer susceptibility candidate 2 (CASC2) for chronic renal failure in patients with type 2 diabetes. Med Sci Monit. 2018;24:6079-84.

14. Fan $Y$, Shi $Y$, Lin Z, Huang $X$, Li J, Huang W, et al. miR-9-5p suppresses malignant biological behaviors of human gastric cancer cells by negative regulation of TNFAIP8L3. Dig Dis Sci. 2019;64(10):2823-9.
15. Wu M, Huang Y, Chen T, Wang W, Yang S, Ye Z, et al. LncRNA MEG3 inhibits the progression of prostate cancer by modulating miR-9-5p/ QKI-5 axis. J Cell Mol Med. 2019;23(1):29-38.

16. Massaro JD, Polli CD, Costa ESM, Alves CC, Passos GA, Sakamoto-Hojo $E T$, et al. Post-transcriptional markers associated with clinical complications in Type 1 and Type 2 diabetes mellitus. Mol Cell Endocrinol. 2019;490:1-14

17. Zhang J, Liu L, Li J, Le TD. LncmiRSRN: identification and analysis of long non-coding RNA related miRNA sponge regulatory network in human cancer. Bioinformatics. 2018;34(24):4232-40.

18. Zhou Z, Wan J, Hou X, Geng J, Li X, Bai X. MicroRNA-27a promotes podocyte injury via PPARgamma-mediated beta-catenin activation in diabetic nephropathy. Cell Death Dis. 2017;8(3):e2658.

19. Duan LJ, Ding M, Hou LJ, Cui YT, Li CJ, Yu DM. Long noncoding RNA TUG1 alleviates extracellular matrix accumulation via mediating microRNA-377 targeting of PPARgamma in diabetic nephropathy. Biochem Biophys Res Commun. 2017;484(3):598-604.

20. Liang W, Sun F. Identification of pivotal IncRNAs in papillary thyroid cancer using IncRNA-mRNA-miRNA ceRNA network analysis. PeerJ. 2019;7:e7441.

21. Li D, Lu Z, Xu Z, et al. Spironolactone promotes autophagy via inhibiting PI3K/AKT/mTOR signalling pathway and reduce adhesive capacity damage in podocytes under mechanical stress. Biosci Rep. 2016;36(4):e00355.

22. Susztak K, Raff AC, Schiffer M, Bottinger EP. Glucose-induced reactive oxygen species cause apoptosis of podocytes and podocyte depletion at the onset of diabetic nephropathy. Diabetes. 2006;55(1):225-33.

23. Chen Z, Ma Y, Yang Q, et al. AKAP1 mediates high glucose-induced mitochondrial fission through the phosphorylation of Drp1 in podocytes. J Cell Physiol. 2020. https://doi.org/10.1002/jcp.29646.

24. Jiang L, Cui H, Ding J. Smad3 signalling affects high glucose-induced podocyte injury via regulation of the cytoskeletal protein transgelin. Nephrology. 2020. https://doi.org/10.1111/nep.13701.

25. Hu M, Wang R, Li X, Fan M, Lin J, Zhen J, et al. LncRNA MALAT1 is dysregulated in diabetic nephropathy and involved in high glucoseinduced podocyte injury via its interplay with beta-catenin. J Cell Mol Med. 2017;21(11):2732-47.

26. Jiao H, Xie D, Qiao Y. LncRNA PRINS is involved in the development of nephropathy in patients with diabetes via interaction with Smad7. Exp Ther Med. 2019;17(4):3203-8.

27. Kim H, Dusabimana T, Kim SR, Je J, Jeong K, Kang MC, et al. Supplementation of abelmoschus manihot ameliorates diabetic nephropathy and hepatic steatosis by activating autophagy in mice. Nutrients. 2018;10(11):1703.

28. Wu F, Li S, Zhang N, Huang W, Li X, Wang M, et al. Hispidulin alleviates high-glucose-induced podocyte injury by regulating protective autophagy. Biomed Pharmacother. 2018;104:307-14.

29. Schmitz KJ, Ademi C, Bertram S, Schmid KW, Baba HA. Prognostic relevance of autophagy-related markers LC3, p62/sequestosome 1, Beclin-1 and ULK1 in colorectal cancer patients with respect to KRAS mutational status. World J Surg Oncol. 2016;14(1):189.

30. Yang H, Kan QE, Su Y, Man H. Long non-coding RNA CASC2 improves diabetic nephropathy by inhibiting JNK pathway. Exp Clin Endocrinol Diabetes. 2019;127(8):533-7.

31. Martinez-Ibarra A, Martinez-Razo LD, Vazquez-Martinez ER, MartinezCruz N, Flores-Ramirez R, Garcia-Gomez E, et al. Unhealthy levels of phthalates and bisphenol A in Mexican pregnant women with gestational diabetes and its association to altered expression of miRNAs involved with metabolic disease. Int J Mol Sci. 2019;20(13):3343.

32. Xiao Y, Guo S, Zhang Y, Bian Z, Jia L, Hu Y, et al. Diabetic nephropathy: serum miR-9 confers a poor prognosis in and is associated with level changes of vascular endothelial growth factor and pigment epithelium-derived factor. Biotechnol Lett. 2017;39(10):1583-90.

33. Zhang Y, Chang B, Zhang J, Wu X. LncRNA SOX2OT alleviates the high glucose-induced podocytes injury through autophagy induction by the miR-9/SIRT1 axis. Exp Mol Pathol. 2019:110:104283. 
34. Higgins LS, Mantzoros CS. The development of INT131 as a Selective PPARgamma Modulator: approach to a safer insulin sensitizer. PPAR Res. 2008;2008:936906.

35. Gandhi GR, Jothi G, Antony PJ, Balakrishna K, Paulraj MG, Ignacimuthu $\mathrm{S}$, et al. Gallic acid attenuates high-fat diet fed-streptozotocin-induced insulin resistance via partial agonism of PPARgamma in experimental type 2 diabetic rats and enhances glucose uptake through translocation and activation of GLUT4 in PI3K/p-Akt signaling pathway. Eur J Pharmacol. 2014;745:201-16.
36. Lee EY, Kim GT, Hyun M, Kim S, Seok S, Choi R, et al. Peroxisome proliferator-activated receptor-delta activation ameliorates albuminuria by preventing nephrin loss and restoring podocyte integrity in type 2 diabetes. Nephrol Dial Transplant. 2012;27(11):4069-79.

\section{Publisher's Note}

Springer Nature remains neutral with regard to jurisdictional claims in published maps and institutional affiliations.
Ready to submit your research? Choose BMC and benefit from:

- fast, convenient online submission

- thorough peer review by experienced researchers in your field

- rapid publication on acceptance

- support for research data, including large and complex data types

- gold Open Access which fosters wider collaboration and increased citations

- maximum visibility for your research: over $100 \mathrm{M}$ website views per year

At BMC, research is always in progress.

Learn more biomedcentral.com/submissions 